\title{
Integrating evidence-based teaching into to clinical practice should improve outcomes
}

\author{
Is evidence-based medicine teaching more effective when integrated into \\ routine practice or when taught in standalone workshops?
}

\section{Coomarasamy A, Khan KS. What is the evidence that postgraduate teaching in evidence based medicine changes anything? A systematic review. Br Med J 2004; 329(7473):1017}

Data sources Sources used were Medline, Embase, the Education Resources Information Centre, Cochrane Controlled Trials Register, Cochrane Database of Systematic Reviews, the Database of Abstracts of Reviews of Effects, Health Technology Assessment database, Best Evidence, Best Evidence Medical Education and Science Citation Index, along with reference lists of known systematic reviews.

Study selection Studies were chosen for inclusion if they evaluated the effects of postgraduate evidence-based medicine (EBM) or critical appraisal teaching in comparison with a control group or baseline before teaching, using a measure of participants' learning achievements or patients' health gains as outcomes.

Data extraction and synthesis Articles were graded as either level 1 (randomised controlled trials (RCT)) or level 2 (non-randomised studies that either had a comparison with a control group), or a before and after comparison without a control group. Learning achievement was assessed separately for knowledge, critical appraisal skills, attitudes and behaviour. Because of obvious heterogeneity in the features of individual studies, their quality and assessment tools used, a metaanalysis could not be carried out. Conclusions were weighted by methodological quality.

Results Twenty-three relevant studies were identified, comprising four $R C T$, seven non-RCT, and 12 before and after comparison studies. Eighteen studies (including two RCT) evaluated a standalone teaching method and five studies (including two RCT) evaluated a clinically integrated teaching method.

Conclusions Standalone teaching improved knowledge but not skills, attitudes or behaviour. Clinically integrated teaching improved knowledge, skills, attitudes and behaviour. Teaching of EBM should be moved from classrooms to clinical practice to achieve improvements in substantial outcomes.

\section{Commentary}

As someone who regularly teaches the evidence-based approach to both undergraduate and postgraduate students it is of great interest to read this systematic review, which focuses on postgraduate education. Although there is increased interest in the teaching of the evidence-based approach in dental schools, with the introduction of problem-based learning and critical appraisal, there is a long way to go before it is fully integrated into school curricula. Consequently these skills will need to be taught at postgraduate level for some time to come: it is useful to know if what we are doing is effective.

Seventeen studies assessed improvement in knowledge. The evidence indicated an improvement from both standalone and integrated methods, the latter changing both attitude and behaviour. Improvements included changes in reading habits and choice of information resources, as well as more important outcomes such as changes in management of patients.

The fact that the integrated approach was able to change behaviour suggests that there is the potential for improving health outcomes. One of the drivers for the evidence-based approach is that clinicians will ultimately adopt more effective treatments, resulting in better outcomes for patients. As the authors point out, however, the translation of changes in behaviour into complex phenomena such as better care of patients may not be a linear one: improving care is likely to be affected by many factors, only one of which will be evidence-based practice. Such changes are also likely to occur over a long period making them difficult to identify.

It is worth noting that all of the studies included were conducted in the medical setting so conclusions may not be directly transferable into a dental environment where different pressures could affect attitudes and behaviours. Consequently more research is still needed in this area.

\section{Practice point}

- Although standalone teaching of evidence-based methods improves knowledge, clinically integrated teaching may result in changes in both attitude and behaviour.

\section{Derek Richards}

Centre for Evidence-based Dentistry, Oxford, UK

Evidence-Based Dentistry (2005) 6, 47.

doi:10.1038/sj.ebd. 6400332 